MIT-CTP 3267

\title{
Gerbes and Duality
}

\author{
M. I. Caicedo ${ }^{12}$ \\ Center for Theoretical Physics, \\ Laboratory for Nuclear Physics and Department of Physics, \\ Massachusetts Institute of Technology \\ Cambridge, Massachusetts 02139 USA \\ and \\ I. Martín ${ }^{3}$ and A. Restuccia円 \\ Departamento de Física \\ Universidad Simón Bolívar. \\ Apartado postal 89000, Caracas 1080-A, Venezuela.
}

\footnotetext{
${ }^{1}$ caicedo@lns.mit.edu also mcaicedo@fis.usb.ve

${ }^{2}$ On sabbatical leave from Departamento de Física, Universidad Simón Bolívar.

3 isbeliam@usb.ve

${ }^{4}$ arestu@usb.ve
} 


\begin{abstract}
We describe a global approach to the study of duality transformations between antisymmetric fields with transitions and argue that the natural geometrical setting for the approach is that of gerbes, these objects are mathematical constructions generalizing $\mathrm{U}(1)$ bundles and are similarly classified by quantized charges. We address the duality maps in terms of the potentials rather than on their field strengths and show the quantum equivalence between dual theories which in turn allows a rigorous proof of a generalized Dirac quantization condition on the couplings. Our approach needs the introduction of an auxiliary form satisfying a global constraint which in the case of 1-form potentials coincides with the quantization of the magnetic flux. We apply our global approach to refine the proof of the duality equivalence between $d=11$ supermembrane and $d=10$ IIA Dirichlet supermembrane.
\end{abstract}

\title{
1 Introduction
}

The usual electromagnetic duality concept first introduced by Dirac in his dissertation on magnetic monopoles, later extended by Montonen and Olive and used lately by Seiberg and Witten [1] to discuss the strong and weak coupling limits of the low energy effective action of $N=2$ SUSY $S U(2)$ YangMills Theory, has provided a breakthrough in the understanding of the non- 
perturbative analysis of QFT. It has also given a powerful tool to unify different superstring and supermembrane theories and to possibly merge them in the context of M-theory, a theory of membranes and 5-branes whose low energy effective action is $d=11$ supergravity [2]. In Maxwell's theory strongweak coupling duality usually referred to as $T$-duality, may be understood as a map between two quantum equivalent $U(1)$ gauge theories, one of them formulated in terms of a $U(1) 1$-form connection $A$ and coupling constant $\tau$ and its dual theory given by another $U(1)$ 1-form connection $V$ and coupling constant $\frac{1}{\tau}$, the dual map being intrinsically non-perturbative [3].

Duality with $p$-forms with $p>1$ was first studied by Barbón in [4] but his results only apply to globally defined $p$-forms. In this article we want to extend those results presenting the most general duality map between locally defined $p$-forms, i.e. antisymmetric fields having non trivial transitions on intersections of the open sets of a covering of a compact $d$-dimensional manifold, in order to achieve this goal, we must introduce the notion of $p$ gerbes. These are geometrical objects which naturally describe the quantized charges associated to antisymmetric fields and are consequently of interest for $D$-brane and $p$-brane theories where the charges have a topological origin.

Gerbes were first introduced by Giraud [5] who was studying non-abelian cohomology. Since then, they have been carefully studied in the mathematical literature [6] [7] [8] [9] unfortunately, only abelian gerbes have been developed into a full geometrical theory so far, while progress in the non 
abelian case although limited and very recent, look quite promising for physical applications [10].

$p$-gerbes are geometrical structures that generalize $U(1)$ principal bundles with connection, in fact, they are the natural setting to allow differential forms to have transitions in much the same way that the $U(1)$ connection does. Gerbes allow the consistent transitioning of $p+1$-order forms by promoting the usual cocycle condition on the intersection of three open sets to a $p+1$-cocycle condition on the intersection of $p+3$ open sets, according to this convention, a line bundle is a 0-gerbe. By costruction, gerbes constitute a sort of "geometrical ladder" in which a line-bundle (0-gerbe) is given by a set of transition functions, a 1-gerbe is given by a set of transition line-bundles, a 2-gerbe is given by a set of transition gerbes, and so on [7] [9]. Gerbeconnections and gerbe-curvatures can be defined by generalization of the corresponding objects for bundles, the curvature of a 0 -gerbe (i.e. of an ordinary connection) is a 2 -form, the curvature of a 1-gerbe is given by a 3 -form the definition obviously extending up the geometrical ladder [12]. These gerbeconnections-curvatures share common issues with line-bundle connections. The Kostant-Weil theorem for example has a gerbe analogue [6] [7], while the first states that line-bundles (0-gerbes) on $\mathcal{M}$ are classified by $H^{2}(\mathcal{M}, \mathbb{Z})$ (the second De Rham integer cohomology over $\mathcal{M}$ ), the latter establishes that equivalence classes of 1 -gerbes on $\mathcal{M}$ are classified by $H^{3}(\mathcal{M}, \mathbb{Z})$, while in general $p$-gerbes are classified by $H^{p+2}(\mathcal{M}, \mathbb{Z}) \cdot p$-gerbe connection have an 
associated notion of parallel transport, the parallel transport of an $p$-gerbe connection is defined along $p+1$ dimensional paths [8] [11] [12]. This in turn brings in the idea of holonomy, in the case ordinary connections on line bundles holonomy associates a group element to each loop while for the case of a 1-gerbe-connection holonomy associates a group element to each 2-loop, some seminal work on this direction was presented in [13].

As we said before, the goal of this work is to study duality maps between $p$-forms with transitions showing that gerbes provide a natural setting for the problem. We shall address the duality in terms not of the field strengths but on their potentials $A_{p}$ and a $d-p-2$ form and will be able to rigourously obtain the generalized Dirac quantization condition on the couplings $g_{p} g_{d-p-2}=2 \pi n$ [14]. To show the quantum equivalence between dual electromagnetic theories, one starts from a theory defined over the space of all connection 1-forms on all line bundles over the base 4-manifold $\mathcal{M}$. The next step in the process consists in building another theory with a Maxwell's like action but in terms of globally constrained 2-forms, at this point another line bundle $\left({ }^{*} L\right)$ is introduced in the game to allow the global contraints to be imposed via Lagrange multipliers. After functional integration of the lagrange multipliers represented by the connection form of ${ }^{*} L$ the dual theory is straightforwardly obtained. In the scheme just described the global constraints are critical since they allow the use of Weil's theorem guaranteeing the existence of a line bundle with connection. The approach may be 
synthesized in the following sequence:

$$
A \Leftrightarrow \Omega_{2}(\text { constrained }) \Leftrightarrow V
$$

where $\Omega_{2}$ is the globally defined constrained 2 -form.

For $p$-forms over gerbes the approach to duality follows similar lines, i.e. duality is shown through a similar sequence of steps which we summarize in the following diagram

$$
A_{p} \Leftrightarrow \Omega_{p+1}(\text { constrained }) \Leftrightarrow V_{d-p-2}
$$

in this latter scheme, $A_{p}$ and $V_{d-p-2}$ represent the dual antisymmetric fields, while $\Omega_{p+1}$ which is constrained to be a closed form with integer periods plays an intermediate role allowing the proof of duality. The constraint on the periods of $\Omega_{p+1}$ is clearly global and consequently it must be implemented ab initio in the mechanism to prove on shell global equivalence and quantum equivalence between dual theories.

For dual maps between 1-form connections in four dimensions the global restriction coincides with the quantization of the magnetic flux. In general, the global condition leads to important relations between the relevant physical parameters involved in the duality map. In the case of the $d=11$ supermembrane $\Leftrightarrow d=10$ Dirichlet supermembrane equivalence, the global constraint becomes the compactification condition on one of the supermembrane coordinates and we can use our machinery to approach this problem. The presentation of the work is as follows, section 2 motivates our general 
programme by carefully reviewing electromagnetic duality emphasizing the role of the above mentioned global constraint. In section 3 we give a brief introduction to gerbes. In section 1 we show that a antisymmetric fields with quantized fluxes naturally give rise to gerbes, this property being of importance for the implementation of our strategy to duality. In section 5 we formulate the general dual map between actions for $p$ and $p-d-2$ forms and show the quantum equivalence of the dual theories. Finally, in section 6 we apply our results to discuss duality maps in $p$-brane theories.

\section{Duality on the space of connections on line bundles}

The purpose of this section is twofold, in the first place, we will construct the quantum formulation of Maxwell's theory in terms of a globally constrained 2form and explicitly show its equivalence to the usual connection formulation. In second place, using the above formulation we will show the duality between two $U(1)$ theories, one of them with coupling constant $\tau$ and the other with coupling $-\frac{1}{\tau}$.

We begin by considering Maxwell's theory, formulated in terms of a con-

nection 1-form $A$ of a $U(1)$ bundle $L$ with base space $\mathcal{M}$-a four dimensional compact orientable euclidean manifold-, the theory is then given by the fol- 
lowing action

$$
I(F(A))=\frac{1}{8 \pi} \int_{\mathcal{M}} d^{4} x \sqrt{g}\left[\frac{4 \pi}{e^{2}} F^{m n} F_{m n}+i \frac{\theta}{4 \pi} \frac{1}{2} \epsilon_{m n p q} F^{m n} F^{p q}\right]
$$

where $(F=d A)$ is the curvature associated to the connection.

Duality is usually addressed in terms of the action of the modular group $S L(2, \mathbb{Z})$ on the complex coupling constant $\tau \equiv \frac{\theta}{2 \pi}+i \frac{4 \pi}{e^{2}}$. Upon introduction of $\tau$ and using the standard decomposition of the curvaturein its self-dual and anti self-dual parts, $I(F(A))$ can be reexpressed as follows

$$
I_{\tau}(A)=\frac{i}{8 \pi} \int_{\mathcal{M}} d^{4} x \sqrt{g}\left[\bar{\tau} F_{m n}^{+} F^{+m n}-\tau F_{m n}^{-} F^{-m n}\right]
$$

or in terms of the inner product of forms

$$
I_{\tau}(A)=\frac{i}{4 \pi}\left[\bar{\tau}\left(F^{+}, F^{+}\right)-\tau\left(F^{-}, F^{-}\right)\right]
$$

We now introduce an similar looking action but now the independent field is a 2 -form $\Omega$ whose only property consisits in being globally defined over $\mathcal{M}$, the action for $\Omega$ is then

$$
\mathcal{I}(\Omega ; \tau)=\frac{i}{4 \pi}\left[\bar{\tau}\left(\Omega^{+}, \Omega^{+}\right)-\tau\left(\Omega^{-}, \Omega^{-}\right)\right]
$$

The quantum field theories associated to actions (2.3) and (2.4) are clearly inequivalent since $\Omega$ is arbitrary, i.e. $\mathcal{I}(\Omega ; \tau)$ is a functional over the whole space of 2-forms, while $F$ in $I_{\tau}(A)$ is the curvature of a $U(1)$ connection.

In order to fulfill our programme of constructing quantum Maxwell's theory in terms of a globally constrained 2-form we will show that after restricting the space of 2 -forms in $\mathcal{I}(\Omega ; \tau)$ by the introduction of two constraints, the 
theories defined by (2.3) and the constrained version of (2.4) are equivalent as QFTs.

The constraints to be imposed on $\Omega$ are

$$
\begin{array}{r}
d \Omega=0 \\
\oint_{\Sigma_{2}} \Omega=2 \pi n
\end{array}
$$

where $\Sigma_{2}$ represents a basis of the integer homology of dimension 2 over $\mathcal{M}$. The first of these constraints restricts $\Omega$ to be closed, while the second ensures its periods to be integers (quantization of the "magnetic flux").

The first step in the discussion is to show that if one introduces a new line bundle -to which we will refer to as the dual line bundle ${ }^{*} L$ - with connection 1-form $V$, it is possible to extend the action $\mathcal{I}(\Omega ; \tau)$ in order to include constraints (2.5) and (2.6) through the appropriate use of $V$ as a Lagrange multiplier in the following way

$$
\mathcal{I}(\Omega, V ; \tau)=\mathcal{I}(\Omega ; \tau)+\frac{i}{2 \pi} \int_{\mathcal{M}} W(V) \wedge \Omega
$$

where, $W(V) \equiv d V$ is the curvature associated to $V$. Before we engage in the rigorous proof of the above claims, we would like to note that using $\int_{\mathcal{M}} W(V) \wedge \Omega=\int_{\mathcal{M}} d V \wedge \Omega$ as the constraint in the extended action $\mathcal{I}(\Omega, V ; \tau)$ instead of the usual formula $\int_{\mathcal{M}} V \wedge d \Omega$ is critical, since as we will show, in the latter case, the constraint in the periods of $\Omega$, which is a global condition, would have never been obtained. 
We begin by considering constraints (2.5) and (2.6). If $F(A)$ is the curvature associated to a connection 1-form then it is obviously closed, i.e. satisfies the local constraint (2.5); moreover, the requirement on the transition functions of the line bundle to be uniform maps over the structure group guarantees that $F(A)$ also satisfies the global constraint on its periods. The following proposition [15] which is a part of the Konstant-Weil theorem, shows that the converse is also true:

If $\Omega$ is a 2-form satisfying constraints (2.5) and (2.0) then there exists a complex line bundle and a connection -not necessarily unique-on it whose curvature is $\Omega$

We will briefly review the proof of the above proposition since we will closely follow it when dealing with higher order $p$-forms. The proof is the following: let $\mathcal{U}=\left\{U_{i}\right\}, i \in I$ ( $I$ a set of indices) be a contractible open covering of $\mathcal{M}$. The condition of closeness on $\Omega$ guarantees that it may be locally expressed as the exterior derivative of a 1-form, in particular, in a triple intersection of open sets $U_{i} \cap U_{j} \cap U_{k} \neq \emptyset, \Omega$ may be written as:

$$
\Omega=d \mathcal{A}_{i}=d \mathcal{A}_{j}=d \mathcal{A}_{k}
$$

where $\mathcal{A}_{j}$ represents an appropriate one form locally defined in $U_{j}$, this in 
turn implies that the local forms must be related by changes given by

$$
\begin{aligned}
& \mathcal{A}_{i}=\mathcal{A}_{j}+d \Lambda_{i j} \\
& \mathcal{A}_{j}=\mathcal{A}_{k}+d \Lambda_{j k} \\
& \mathcal{A}_{k}=\mathcal{A}_{i}+d \Lambda_{k i}
\end{aligned}
$$

where now the $\Lambda$ 's are local 0 -forms. This last set of identities lead to conclude that

$$
\Lambda_{i j}+\Lambda_{j k}+\Lambda_{k i}=\text { constant }=c
$$

Finally, the global condition on the periods of $\Omega$ leads to (see section 4 for details),

$$
c=2 \pi n
$$

The conclusion of these steps is clearly that in the sense of Čech the cochain 16]

$$
g:(i, j) \rightarrow g_{i j} \equiv e^{i \Lambda_{i j}} \in U(1)
$$

is a 1-cocycle

$$
\delta g_{i j k}=g_{i j} g_{j k} g_{k i}=1
$$

Moreover, if the local 1 -forms are changed in intersecting open sets by gauge transformations

$$
\begin{aligned}
& \mathcal{A}_{i} \rightarrow \mathcal{A}_{i}+d \lambda_{i} \text { in } U_{i} \\
& \mathcal{A}_{j} \rightarrow \mathcal{A}_{j}+d \lambda_{j} \text { in } U_{j}
\end{aligned}
$$


the glueing 0 -forms must change as

$$
\Lambda_{i j} \rightarrow \Lambda_{i j}+\lambda_{i}-\lambda_{j}
$$

implying that $g_{i j}$ changes as

$$
g_{i j} \rightarrow h_{i} g_{i j} h_{j}^{-1} .
$$

One then notice that $h_{i} h_{j}^{-1}$ is a coboundary as follows from the fact that

$$
h:(i) \rightarrow h_{i}=e^{i \lambda_{i}} \in U(1)
$$

is a map from $U_{i}$ to the structure group, and

$$
\delta h(i, j)=h_{i} h_{j}^{-1}
$$

consequently, under (2.18) $g_{i j}$ changes by a coboundary, and then after the change it still defines the same element of the Cech cohomolog $\check{H}^{1}\left(\mathcal{U}, \mathbb{C}^{*}\right)$, where $\mathbb{C}^{*}$ is the set of non zero complex numbers. It is known 16 that there is a one-to-one correspondence between $\check{H}^{1}\left(\mathcal{U}, \mathbb{C}^{*}\right)$ and the complex line bundles over $\mathcal{M}, g_{i j}$ defining the transition functions of the bundle, therefore, constraints (2.5) and (2.6) then define an unique line bundle over $\mathcal{M}$. Moreover $\mathcal{A}$, defined by patching together the 1 -forms $\mathcal{A}_{i}$ by using (2.16)(2.17), is a connection 1-form over $\mathcal{M}$ and $\Omega$ its curvature 2 -form.

Regarding the non uniqueness of the connections on the line bundle associated to $\Omega$, one must realize that two connection 1-forms $\mathcal{A}^{(1)}$ and $\mathcal{A}^{(2)}$ with the same curvature may be in different equivalence classes not related by 
gauge transformations. They differ at most by a closed 1-form $\theta \in H^{1}(\mathcal{M}, \Re)$. If $\theta$ is an element of $H^{1}(\mathcal{M}, \mathbb{Z})$ then $\mathcal{A}^{(1)}$ and $\mathcal{A}^{(2)}$ are connections on the same equivalence class but otherwise they belong to different ones. The equivalence classes of connections related to the same $\Omega$ are in one-to-one correspondence to $H^{1}(\mathcal{M}, U(1))$. Moreover, one has for the holonomy maps $Q$ constructed with connections with the same curvature $\Omega$,

$$
Q^{\chi \cdot l}=\chi Q^{l}
$$

here $l$ denotes a line bundle with a particular equivalence class of connections and $\chi$ is the holonomy map given by the exponential of the integral of $\theta$ around a closed curve. For a simple connected base manifold $\mathcal{M}$ the line bundle associated to $\Omega$ is unique [15].

The observation just made is relevant to the proof of the quantum equivalence of the theories defined by $I_{\tau}(A)$ and $\mathcal{I}(\Omega)$ restricted by the constraints we have been studying. Indeed, when formulating the quantum correlation functions for either theory, one must carefully define the functional measure in order to account for the "zero modes", that is the space $H^{1}(\mathcal{M}, \Re)$.

It is worth noticing that -up to the definition of the measure-, the equivalence of the quantum theories rests on the non local constraint on the periods of the 2-form $\Omega$. Indeed, since the local restriction $d \Omega=0$ is not sufficient to guarantee the existence of a line bundle and a connection with curvature $\Omega$ there is no local formulation of Maxwell's theory $\left(I_{\tau}(A)\right)$ in terms of a globally defined closed 2-form $\Omega$. And therefore, the global constraint that 
associates a set of integers $\{n\}$ (the winding numbers or topological charges) to the elements of a basis of homology of dimension 2 to $\Omega$ is a must.

In order to continue with the proof of the quantum equivalence, we come to study the formulation of the off shell Lagrange problem associated to action (2.4), and constraints (2.5) and (2.6). We will show that action $\mathcal{I}(\Omega ; \tau)$ constrained by both the local $(d \Omega=0)$ and global $\left(\int \Omega=2 n \pi\right)$ conditions and the extended action $\mathcal{I}(\Omega, V ; \tau)$ are equivalent quantum mechanically when summation over all line bundles is considered in the functional integral.

We first consider the extra piece in $\mathcal{I}(\Omega, V)$ i.e.

$$
\mathcal{S}_{\text {Lagrange }}=\frac{i}{2 \pi} \int_{\mathcal{M}} d V \wedge \Omega
$$

where we must recall that $V$ is a connection 1-form on the dual bundle ${ }^{*} L$. $\mathcal{S}_{\text {Lagrange }}$ can be rewritten as

$$
\mathcal{S}_{\text {Lagrange }}=-\frac{i}{2 \pi} \int_{\mathcal{M}} V \wedge d \Omega+\frac{i}{2 \pi} \int_{\mathcal{M}} d(V \wedge \Omega)
$$

The functional integration on $V$ may be performed in two steps. We first integrate on all connections over a given complex line bundle and then over all complex line bundles. The second term on (2.23) depends only on the transition function of a given complex line bundle, while the first depends also on the space of connections over the line bundle. Integration associated to the first step yields the following factor

$$
\delta(d \Omega)
$$


on the functional measure.

It is convenient to rewrite the second term in $(2.23)$ as

$$
\begin{aligned}
\frac{i}{2 \pi} \int_{\mathcal{M}} d(V \wedge \Omega) & =\frac{i}{2 \pi} \int_{\Sigma_{3}}\left(V_{+}-V_{-}\right) \wedge \Omega= \\
& =\frac{i}{2 \pi} \int_{\Sigma_{3}} d \xi_{+-} \wedge \Omega=i n \int_{\Sigma_{2}} \Omega
\end{aligned}
$$

where $\Sigma_{3}$ stand for 3-dimensional surfaces living in the intersection of open sets where the transition of the connection 1-form $V$ takes place,

$$
\begin{aligned}
V_{+}-V_{-} & =d \xi_{+-} \\
g_{+-} & =e^{i \xi_{+-}}
\end{aligned}
$$

$g_{+-}$being the transition function and $\xi_{+-}$is, in general, a multivalued function.

Recalling that the summation must be over all line bundles one finds that formula (2.25) brings in the following factor to the measure of the path integral

$$
\sum_{m} \delta\left(\int_{\Sigma_{2}} \Omega-2 \pi m\right)
$$

where $\Sigma_{2}$ denotes a basis of an integer homology of dimension 2. We thus conclude that the Lagrange problem associated to the action (2.4) constrained by (2.5) (2.6) is indeed given by the extended action (2.7).

We now turn to the discussion of the full partition function associated to the extended action $\mathcal{I}(\Omega, V ; \tau)$. The path integral that we want to calculate 
is given by

$$
\mathcal{Z}(\tau)=\sum_{m} \int \mathcal{D} \Omega \mathcal{D} V \operatorname{Vol}_{Z M} \operatorname{det}\left(d_{2}\right) \frac{1}{\operatorname{Vol} G} e^{-\mathcal{I}(\Omega, V ; \tau)}
$$

where as we have just learned, $\sum_{m}$ stands for summation over all line bundles. $\operatorname{Vol}_{Z M}$ is the volume of the space $H^{1}(\mathcal{M}, R)$, $\operatorname{det}\left(d_{2}\right)$ is the determinant of the exterior differential operator on 2-forms and $\operatorname{Vol} G$ is the volume of the gauge group. After performing the integration on $V$ as described in the previous paragraphs, we obtain

$$
\mathcal{Z}(\tau)=\sum_{m} \int \mathcal{D} \Omega \operatorname{Vol}_{Z M} \operatorname{det}\left(d_{2}\right) \delta(d \Omega) \delta\left(\int_{\Sigma_{2}} \Omega-2 \pi m\right) e^{-\mathcal{I}(\Omega ; \tau)}
$$

The measure may now be reexpressed in terms of an integration on the space of connections $A$ over the line bundle $L$ in the following way

$$
\mathcal{Z}(\tau)=\sum_{m} \int \mathcal{D} \Omega \operatorname{Vol}_{Z M} \int \mathcal{D} A \frac{1}{\operatorname{Vol} G} \frac{\delta(\Omega-F(A))}{\operatorname{Vol}_{Z M}} \delta\left(\int_{\Sigma_{2}} \Omega-2 \pi m\right) e^{-\mathcal{I}(\Omega ; \tau)}
$$

The factor $1 / \mathrm{Vol}_{Z M}$ that comes from reexpressing $\delta(d \Omega)$ in terms of $\delta(\Omega-F(A))$ exactly cancels the volume originally appearing in the functional measure. Further integration in $\Omega$ produces the final result

$$
\mathcal{Z}(\tau)=\mathcal{N} \int \hat{\mathcal{D}} A \frac{1}{\operatorname{Vol} G} e^{-I_{\tau}(A)}
$$

Where $\hat{\mathcal{D}} A$ denotes integration over the space of connections on all line bundles over $\mathcal{M}$. Since (2.32) is the partition function for the action $I_{\tau}(A)$, we have been able to show the quantum equivalence of the three formulations of Maxwell's theory thus finishing the first part of our programme. 
Finally, we would like to briefly discuss the duality transformations in the functional integral associated to Maxwell's theory. We start from the action

$$
\begin{aligned}
& \mathcal{I}(\Omega, V)=\frac{i}{4 \pi}\left[\bar{\tau}\left(\Omega^{+}, \Omega^{+}\right)-\tau\left(\Omega^{-}, \Omega^{-}\right)\right] \\
& +\frac{i}{2 \pi}\left(W^{+}(V), \Omega^{+}\right)+\frac{i}{2 \pi}\left(W^{-}(V), \Omega^{-}\right),
\end{aligned}
$$

from where it is possible to perform the functional integration on $\Omega^{+}$and $\Omega^{-}$ to get the known result [3]

$$
\mathcal{Z}(\tau)=\mathcal{N} \tau^{-\frac{1}{2} B_{2}^{-}} \bar{\tau}^{-\frac{1}{2} B_{2}^{+}} \mathcal{Z}\left(-\frac{1}{\tau}\right)
$$

where $B_{k}^{+}$and $B_{k}^{-}$are the dimensions of the spaces of selfdual and antiselfdual $k$ forms, this last formula can be reexpressed in terms of the Euler characteristic $\chi$ and the Hirzebruch signature $\sigma$ as

$$
\left[\operatorname{Im}(\tau)^{\frac{1}{2}\left(B_{0}-B_{1}\right)} \mathcal{Z}(\tau)\right]=\mathcal{N} \tau^{-\frac{1}{4}(\chi-\sigma)} \bar{\tau}^{-\frac{1}{4}(\chi+\sigma)}\left[\operatorname{Im}\left(-\frac{1}{\tau}\right)^{\frac{1}{2}\left(B_{0}-B_{1}\right)} \mathcal{Z}\left(-\frac{1}{\tau}\right)\right.
$$

$\mathcal{N}$ is a factor independent of $\tau$ that depends on the topology of $\mathcal{M}$.

We have thus been able to implement the duality transformations in a rigorous way by including the global constraint and the associated measure factors in the functional integral of the Maxwell action over a general base manifold $\mathcal{M}$.

\section{$3 \quad$ Introducing Gerbes}

In most of the standard literature, antisymmetric tensors fields are described in terms of global $p$-form potentials defined over a manifold $\mathcal{M}$, since these 
forms are global they don't transition as usual connections do and therefore it is not clear how they may adequately describe generalizations of magnetic monopoles. In order to describe antisymmetric fields in the most general way fields with nontrivial transitions over the base manifold must be accounted for, their "curvature" being a globally defined $p+1$-form. These transitioning field configurations are the ones responsible for the appearance of topological charges, this latter observation being essential in the description of $p$-branes and $D$-branes from a quantum field theory point of view. The natural setting for the description of the above mentioned $p$-form potentials is in terms of $p$-gerbes which we will try to describe in this section. Recalling that, as we mentioned in the introduction, gerbes constitute a sort of geometrical ladder (a 0-gerbe for instance being nothing but a line bundle $L$ ) we will try to introduce gerbes by describing the lowest steps in such ladder.

The simplest way to define gerbes is by specifying the data which is needed to reconstruct them. To build a 0-gerbe all that is needed is a contractible open covering $\mathcal{U}$ of $\mathcal{M}$ and a set of transition functions $g_{i j}: U_{i} \cap U_{j} \rightarrow U(1)$ satisfying the usual rules: $g_{i i}=1, g_{i j}=g_{j i}^{-1}$ and $g_{i j} g_{j k} g_{k i}=1$ for any nonempty triple intersection, the last rule obviously being the Čech condition for a 1-cocycle.

The next objects in the hierarchy are 1-gerbes. Their defining data are [6] [9] an open covering of $\mathcal{M}$, and a set of maps

$$
g_{i j k}: U_{i} \cap U_{j} \cap U_{k} \rightarrow S^{1}
$$


defined on each triple intersection satisfying

$$
g_{i j k}=g_{j i k}^{-1}=g_{i k j}^{-1}=g_{k j i}^{-1}
$$

and a 2-cocycle condition, in the intersection of four open sets, namely

$$
\delta g=g_{j k \ell} g_{i k \ell}^{-1} g_{i j \ell} g_{i j k}^{-1}=1 \quad \text { on } \quad U_{i} \cap U_{j} \cap U_{k} \cap U_{\ell}
$$

Similar definitions apply for higher order $p$-gerbes, i.e. their data are given by $S_{1}$ valued maps $g_{i_{0} i_{1} \ldots i_{p}}$ defined on the intersection of $p+2$-sets and which satisfy a $p+1$-cocycle condition on the intersection of $p+3$ open sets.

Gerbes are sufficiently well behaved objects as to allow differential geometry, gerbe-connections and gerbe-curvatures can be defined by properly generalizing the corresponding objects for bundles. The curvature of a 0gerbe is obviously the curvature 2 -form of the bundle, the curvature of a 1-gerbe is given by a closed 3-form and in general the curvature of a $p$-gerbe is a $p+2$ closed form. These closed forms are the natural descendants of a tower of differential forms (of orders 0 to $p+1$ ) which, as we shall see in this section, do naturally define the gerbes and which are thus referred to as the gerbe connection. $p$-gerbe connection have an associated notion of parallel transport, the parallel transport of an p-gerbe connection is defined along $p+1$ dimensional paths [8] [11][12] and allow to define holonomies, which in the case ordinary connections on 0 -gerbes associate an element of $U(1)$ to each loop, while for the case of a 1-gerbe-connection the association is form 2-loops to the group. In any case, these parallel transport holonomy notios 
for $p$-gerbes call in the use of categories[12] and are not of concern for this work.

One particularly interesting feature of gerbes is that in general they are not manifolds, consequently we cannot point to them as spaces as we do with line bundles. Fortunately, the notion of a trivialization of a gerbe gives some insight about these objects [9]. In the case of a 0-gerbe (a line bundle $L$ ), a trivialization is a non-vanishing section $s$ of $L$. If one choses $s$ to be unitary it is also a section of a principal $S^{1}$-bundle, i.e. a collection of maps $f_{i}: U_{i} \rightarrow S^{1}$ where the open sets $U_{i}$ for a covering of the base space and which in any intersection $U_{i} \cap U_{j}$ satisfies the rules

$$
f_{i}=g_{i j} f_{j}
$$

where the $g_{i j}$ are the transition maps for the bundle. Given a different trivialization $f_{i}^{\prime}$ the difference between the two trivializations is defined as the family of quotients $f_{i}^{\prime} / f_{i}$. By construction one finds that

$$
\frac{f_{i}^{\prime}}{f_{i}}=\frac{f_{j}^{\prime}}{f_{j}}
$$

which shows that $f_{i}^{\prime} / f_{i}$ is the restriction to $U_{i}$ of a global map thus showing that for a 0-gerbe the difference between two trivializations is nothing but a global map $F: \mathcal{M} \rightarrow S^{1}$.

For 1-gerbes a trivialization is defined by a set of functions

$$
f_{i j}=f_{j i}^{-1}: U_{i} \cap U_{j} \rightarrow S^{1}
$$


such that $g_{i j k}=f_{i j} f_{j k} f_{k i}$. Once again, the difference between two trivializations is given by the quotients $h_{i j}=f_{i j}^{\prime} / f_{i j}$, which in turn satisfy

$$
h_{i j} h_{j k} h_{k i}=1
$$

this is no other but Čech condition for a 1-cocycle, meaning that the difference between two trivializations of a 1-gerbe is a line bundle.

To make the definition of gerbes rigorously complete we should mention that there must be some independence on the choice of the trivializations. For 0-gerbes this is reflected on the fact that a line bundle is an element of $\breve{H}^{1}(X, \Re)$, i.e. an equivalence class of 1-Čech cocycles. Given the proper definition of equivalence, 1-gerbes are bijectively related to $\check{H}^{2}\left(\mathcal{M}, \underline{U}_{\mathcal{M}}(1)\right)$ [9]. In the general case the generalization of the notion of equivalence calls for the use of categories, for our current purposes we won't go into such details but rather refer to the mathematical literature [6] 9].

An alternative -much clearer and closer to the physicist- picture of gerbes can be given in terms of multiplets of forms, and can be conveniently introduced, risking being reiterative, by briefly reviewing the field theory formulation of 1-form connections or stated in other words, the theory of line bundles formulated in terms of their connections.

The isomorphism classes of line bundles with connections may be represented by equivalence classes of doublets $(A, g)$ where $A$ is a 1 -form connection over the base manifold $\mathcal{M}$ and $g$ are the transition functions with values in $\mathbb{C}^{*}$-the nonzero complex numbers-. The connection (potential) is 
built form a set of local one forms $\left(A_{i}\right)$ which on double intersections must be related by the transitions as usual, i.e. $A_{i}-A_{j}=g_{i j}^{-1} d g_{i j}$. The transition maps must satisfy the standard conditions $g_{i i}=I, g_{i j}=g_{j i}^{-1}$ and the cocycle condition $(\delta g)_{i j k}=g_{i j} g_{j k} g_{k i}=I$ which in terms of the exponential (or $\log$ ) map is usually cast as $\Lambda_{i j}+\Lambda_{i j}+\Lambda_{i j}=2 \pi n, n \in Z$

The equivalence relation between to doublets is defined by stating that two doublets are equivalent or gauge equivalent in physicists language $\left(\left(A^{\prime}, g^{\prime}\right) \sim\right.$ $(A, g))$ if and and only if, for any $U_{i}$ there is a map $h_{i}: U_{i} \rightarrow \mathbb{C}^{*}$ such that the potentials and the transition functions are related in the usual way as $A_{i}^{\prime}=A_{i}+h_{i}^{-1} d h_{i}$ and $g_{i j}^{\prime}=h_{i} g_{i j} h_{j}^{-1}$ on $U_{i} \cap U_{j}$, this relations are much more familiar if we express them in terms of the arguments of the exponential map, which formulates the transitions as in formulas (2.16), (2.17) and (2.18). In such representation, the equivalence between the transition 0-forms shows that the integer appearing in formula (2.12) classifies the doublet, the integer being the quantized flux $\int F / 2 \pi \in H^{2}(\mathcal{M}, \mathbb{Z})$. This is just the fact that line bundles are classified by second integer De Rham cohomology over $\mathcal{M}\left(H^{2}(\mathcal{M}, \mathbb{Z})\right)$ which comes from the isomorphism $\check{H}^{1}\left(\mathcal{M}, \underline{U}_{\mathcal{M}}(1)\right) \cong H^{2}(\mathcal{M}, \mathbb{Z})$ which in turn follows from the exact sequence of sheaves

$$
0 \longrightarrow \mathbb{Z}_{M} \stackrel{\times 2 \pi i}{\longrightarrow} \mathbb{C}_{M} \stackrel{\exp }{\longrightarrow} \mathbb{C}_{M}^{*} \longrightarrow 1
$$

and the isomorphism $\check{H}^{2}\left(\mathcal{M}, \mathbb{Z}_{\mathcal{M}}(1)\right) \cong H^{2}(\mathcal{M}, \mathbb{Z})$ [6] [12].

Let us now describe the generalization of the doublet structure to a set 
of triplets $(B, \eta, \Lambda)$, where $B$ represents a 2 -form locally defined on the open sets of a covering, $\eta$ is a local 1 -form defined on double intersections, and $\Lambda$ is a 0 -form -the transition function for the 1-forms- locally defined on triple intersections (The generalization to $p$-plets goes along the same lines).

Let $B_{i}$ stand for the local 2-form as described in $U_{i}$, an element of an open covering of $\mathcal{M}$, in analogy with the connection one forms these 2 -forms must transition on the intersection of two open sets, the only difference is that now the transitions are given by the 1-forms of the multiplet as

$$
B_{i}-B_{j}=d \eta_{i j}
$$

the 1-forms $\eta_{i j}$ in turn must be matched (must transition) on the intersection of three open sets i.e. when $U_{i} \cap U_{j} \cap U_{k} \neq \emptyset$

$$
\eta_{i j}+\eta_{j k}+\eta_{k i}=d \Lambda_{i j k}
$$

while on $U_{i} \cap U_{j} \cap U_{k} \cap U_{l} \neq \emptyset$, the 0 forms $\Lambda$ satisfy the 2-cocycle condition

$$
(\delta \Lambda)_{i j k l}=\Lambda_{i j k}-\Lambda_{i j l}+\Lambda_{i k l}-\Lambda_{j k l}=2 \pi n .
$$

(3.44), (3.45) and (3.46) define the transition properties of a triplet $(B, \eta, \Lambda)$ on a covering of $\mathcal{M}$. To give a complete description of the triplet defining a 1-gerbe we need to introduce a notion of the equivalence between triplets.

Two triplets $(B, \eta, \Lambda)$ and $(\tilde{B}, \tilde{\eta}, \tilde{\Lambda})$ if for each open set $U_{i}$ and each nonempty intersection $U_{i} \cap U_{j} \neq \emptyset$ there exists 1-forms $\omega_{i}$ and 0 -forms $\Theta_{i j}$ 
such that the local forms in each triplet are related by

$$
\begin{aligned}
\hat{B}_{i} & =B_{i}+d \omega_{i} \\
\hat{\eta}_{i j} & =\eta_{i j}+\omega_{i}-\omega_{j}+d \theta_{i j} \\
\hat{\Lambda}_{i j k} & =\Lambda_{i j k}+\theta_{i j}+\theta_{j k}+\theta_{k i}
\end{aligned}
$$

rules (3.48), (3.49) and (3.49) define the gauge transformations on the space of triplets and clearly generalize those of the connection and transition functions for a line bundle (0-gerbe). With these definitions the equivalence classes of triplets are independent of the covering, and the isomorphism $\check{H}^{1}\left(\mathcal{M}, \underline{C}_{\mathcal{M}}^{*}\right) \cong \check{H}^{2}(\mathcal{M}, \mathbb{Z})$ generalizes to the following isomorphism between cohomology groups

$$
\check{H}^{2}\left(\mathcal{M}, \underline{C}_{\mathcal{M}}^{*}\right) \cong \check{H}^{3}(\mathcal{M}, \mathbb{Z})
$$

where the second Čech cohomology group $\check{H}^{2}\left(\mathcal{M}, \underline{C}_{\mathcal{M}}^{*}\right)$ is to be identified with the 0 -form transitions satisfying the 2-cocycle condition, and $\check{H}^{3}(\mathcal{M}, \mathbb{Z})$ is related but not exactly isomorphic to the 3 -forms with integer periods, and in fact it generalizes the Chern classes of doublets to triplets.

The identification between gerbes and multiplets of fields was first suggested by Deligne [6] [12], in the case of 0-gerbes, the equivalence classes of line bundles with connection are in one-to-one correspondence to the cohomology classes in the first smooth Deligne hypercohomology group:

$$
H^{1}\left(\mathcal{M}, \underline{\mathbb{C}}_{\mathcal{M}}^{*} \stackrel{\text { dlog }}{\longrightarrow} \underline{\mathcal{A}}_{\mathcal{M}, \mathbb{C}}^{1}\right)
$$


while for triplets (1-gerbes with connection) the bijective correspondence is to the second cohomology class of Deligne's hypercohomology

$$
H^{2}\left(\mathcal{M}, \underline{\mathbb{C}}_{\mathcal{M}}^{*} \stackrel{\text { dlog }}{\longrightarrow} \underline{\mathcal{A}}_{\mathcal{M}, \mathbb{C}}^{1} \stackrel{d}{\longrightarrow} \underline{\mathcal{A}}_{\mathcal{M}, \mathbb{C}}^{2}\right)
$$

These and other relevant exact sequences have been extensively studied in [9] and the important conclusion is that in general $p$-gerbes are classified by $H^{p+2}(\mathcal{M}, \mathbb{Z})[12]$.

\section{Flux quantization and Gerbes}

The purpose of this section is to show how a closed form with a quantized flux naturally describes a gerbe in much the same way that a magnetic monopoles describes a bundle with connection. In a sense, we will be generalizing the reasoning behind Weil's theorem. Before entering the subject we will introduce a unified notation, a one indexed object (such as $A_{2}$ ) stands for a 2 form, while something like $\Lambda_{1 i}$ is a 1-form locally defined on an element $U_{i}$ of a covering.

We consider closed integer forms globally defined over $\mathcal{M}$-an orientable compact euclidean manifold- and explicitly show that such forms have a natural geometrical structure associated them. This geometrical structure consists of equivalence classes of multiplets of local forms generalizing the local connection 1-forms and their related transition functions. For a $p+$ 1-form $F_{p+1}$, the multiplet contains local $p$-forms with values in the $U(1)$ 
algebra, and a tower of forms of decreasing order which ends up in zero forms satisfying a $p$-cocycle condition in the intersection of $p+2$ open neighborhoods of a covering of $\mathcal{M}$ and thus the strucutre is that of a $p-2$-gerbe. In the particular case $p=3$ for example, a closed 3 form $F_{3}$ with integer flux gives rise to triplets $\left(A_{2 i}, \Lambda_{1 i}, \Lambda_{0 i}\right)$ of local forms defined in each open set $\mathcal{U}_{i}$, i.e. a 1-gerbe.

For the sake of completeness, we start our presentation with the simplest case: $p=1$, this is relevant to show the equivalence between the $d=11$ supermembrane and the $d=10$ IIA Dirichlet supermembrane which we will adress in the next section.

To begin our discussion, let $F_{1}$ be a 1 -form globally defined over $\mathcal{M}$ satisfying

$$
\begin{aligned}
d F_{1} & =0 \\
\oint_{\Sigma_{1}} F_{1} & =2 \pi n, \quad n \in \mathbb{Z}
\end{aligned}
$$

where $\Sigma_{1}$ is a basis of an integer homology of curves over $\mathcal{M}$ and $n$ is an integer associated to each element of the basis, then $F_{1}$ must be given by

$$
F_{1}=-i g^{-1} d g
$$

where

$$
g=\exp i \varphi
$$


defines an uniform map

$$
\mathcal{M} \rightarrow S^{1},
$$

$\varphi$ being an angular coordinate on $S^{1}$.

Conversely, given $g$ an uniform map from $\mathcal{M} \rightarrow S^{1}$ then the formula $-i g^{-1} d g$ does indeed define a closed 1-form with integer periods. To prove this claim, we begin by realizing that with $F_{1}$ given as in (4.55), one may define

$$
\varphi(P)=\int_{O}^{P} F_{1}
$$

where $O$ and $P$ are the two end points of a curve on the base manifold $\mathcal{M}, O$ being a reference point. $\varphi(P)$ is independent of the curve within a homology class in the sense that if $\mathcal{C}$ and $\mathcal{C}^{\prime}$ are open curves with the same end points

$$
\int_{\mathcal{C}} F_{1}=\int_{\mathcal{C}^{\prime}} F_{1}
$$

if the closed curve $\mathcal{C}^{-1} \mathcal{C}^{\prime}$ is homologous to zero and, by assumption 4.54, differs in $2 \pi n$ between two different homology classes. (1.56) then defines an uniform map from $\mathcal{M} \rightarrow S^{1}$. The converse follow directly by the same arguments.

We may also understand the origin of $\varphi$ as given in (4.58) from a different point of view by considering a covering of $\mathcal{M}$ with open sets $U_{i}, i \in I$. We may always assume $U_{i}$ and $U_{i} \cap U_{j} \neq \emptyset, i, j \in I$ to be contractible to a point.

Since $F_{1}$ is closed it is locally exact, and thus on $U_{i}, i \in I$

$$
F_{1}=d \lambda_{0 i} \quad \lambda_{0 i} \text { being a local 0-form, }
$$


and on $U_{i} \cap U_{j} \neq \emptyset$

$$
\begin{aligned}
d \lambda_{0 i} & =d \lambda_{0 j} \\
\lambda_{0 i} & =\lambda_{0 j}+c_{j i}
\end{aligned}
$$

where $c_{j i}$ is a constant on the intersection.

On $U_{j}$ we may define

$$
\lambda_{0 j}^{\prime}=\lambda_{0 j}+c_{j i}
$$

without changing $F_{1}$ and with a trivial transition on $U_{i} \cap U_{j} \neq \emptyset$.

We may try continue this process of redefining $\lambda_{0 i}$ through trivial transitioning in order to systematically extend it to all the open sets on the covering, but at some point $U_{j}$, the global condition on the periods of $F_{1}$ will impose an obstruction to the process, and then we will only be able to write

$$
F_{1}=d \lambda
$$

at the expense of dealing with a multivalued function $\lambda$. Condition (4.54) thus ensures that the transition (which in this case defines the multivaluedness of $\lambda$ ) is $2 \pi n$, and we do consequently obtain (4.55), (4.56) and (4.57).

Let us consider the degree 2 case already discussed in section 2 to which we would like to add some remarks. Let $F_{2}$ be a 2 -form globally defined on $\mathcal{M}$ and let $F_{2}$ be closed and satisfy the constraint on its periods $\left(\oint_{\Sigma_{2}} F_{2}=2 \pi n\right)$ on a basis of an integer homology of dimension $2\left(\Sigma_{2}\right)$. Then according to 
the Konstant-Weil theorem there exists a complex line bundle with conection with base space $\mathcal{M}$ whose curvature is $F_{2}$ and conversely, given a line bundle over $\mathcal{M}$ with local connection 1-form $A_{1 i}$ its curvature $F_{2}=d A_{1 i}$ satisfies is both closed and integer.

We are interested in the first statement, cuantized flux implies the existence of a 0-gerbe. As we showed in section 2 iterative use of Poincarè's lemma shows that in each open set of a covering of $\mathcal{M} F_{2}$ is given by a set of local 1-form $A_{1 i}$, which on nonempty intersections of two elements of the covering must be related by transition 0 -forms in the usual fashion $A_{1 i}=A_{1 j}+d \Lambda_{0 i j}$ the transition 0-forms in turn being forced to satisfy the condition: $\Lambda_{0 i j}+\Lambda_{0 j k}+\Lambda_{0 k i}=$ constant on triple not empty intersections $\left(U_{i} \cap U_{j} \cap U_{k} \neq \emptyset\right)$. We will now complete a detail that we left open in section 2, the proof that the constant in the latter condition is given by the period of $F_{2}$, indeed, if we take a "surface" $\Sigma_{2}$ in the intersection $U_{i}, U_{j}$ and $U_{k}$, (see Figure 1), we can calculate the period of $F_{2}$ to get

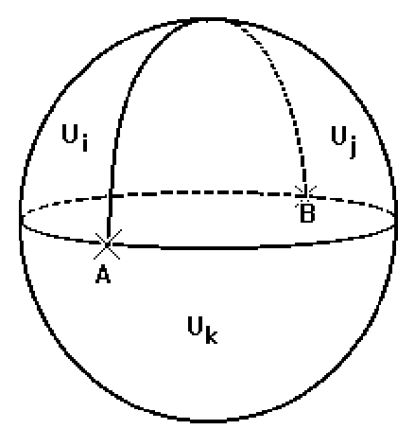

Figure 1: Three intersecting open sets 


$$
\int_{\Sigma_{2}} F_{2}=\int_{\Sigma_{1}} A_{1}=\left.\left(\Lambda_{0 i j}+\Lambda_{0 j k}+\Lambda_{0 k i}\right)\right|_{B} ^{A}=2 \pi n
$$

where $\Sigma_{1}$ is the union of the three curves on the figure, and $A_{1}=d\left(\Lambda_{0 i j}+\right.$ $\left.\Lambda_{0 j k}+\Lambda_{0 k i}\right)$. Without loosing generality we may redefine the $\Lambda$ 's in such a way as to make the value of $\Lambda_{0 i j}+\Lambda_{0 j k}+\Lambda_{0 k i}$ at $B$ equal to zero, and thus obtain the cocycle condition

$$
\Lambda_{0 i j}+\Lambda_{0 j k}+\Lambda_{0 k i}=2 \pi n
$$

To complete the construction of the 0 -gerbe associated to $F_{2}$ equivalence classes of doublets of forms $\left(A_{1}, \Lambda_{0}\right)$ must be adequately defined. As we already know, this is done by introducing local 0 -forms $\lambda_{0 i}$ and declaring $\left(A_{1}, \Lambda_{0}\right) \sim\left(\tilde{A}_{1}, \tilde{\Lambda}_{0}\right)$ if $\tilde{\Lambda}_{0 i j}-\Lambda_{0 i j}=\lambda_{0 i}-\lambda_{0 j}$ and $\tilde{A}_{1 i}-A_{1 i}=d \lambda_{0 i}$

In what follows we will try to follow the steps we have just taken, i.e. an iterative procedure involving Poincarès's lemma, in order to build similar multiplets of forms for higher order closed and globally defined forms. With this in mind, let us now consider a 3 -form $F_{3}$ globally defined on $\mathcal{M}$ satisfying the constraints we have been considering, i.e. closedness and the "quantization" of its flux,

$$
d F_{3}=0 \quad \text { and } \quad \oint_{\Sigma_{3}} F_{3}=2 \pi n, \quad n \in \mathbb{Z}
$$

where $\Sigma_{3}$ is a basis of an integer homology of dimension 3 .

Since $F_{3}$ is closed we can build a local 2 form $A_{2 i}$ in any subset $U_{i}$ of the covering in such a way as to have

$$
F_{3}=d A_{2 i}
$$


and since in the intersection of two sets $\left(U_{i} \cap U_{j} \neq \emptyset\right)$

$$
d A_{2 i}=d A_{2 j}
$$

the local 2 forms must by related (transition) as

$$
A_{2 i}=A_{2 j}+d \Lambda_{1 i j}
$$

where this time, the transition is given by, a 1 -form $\Lambda_{1 i j}$ which is locally defined on $U_{i} \cap U_{j}$. Clearly, on a nonempty triple intersection $U_{i} \cap U_{j} \cap U_{k}$ it happens that

$$
d\left(\Lambda_{1 i j}+\Lambda_{1 j k}+\Lambda_{1 k i}\right)=0
$$

meaning that in such triple intersection the sum of the transition one forms is locally a 0 -form, i.e.

$$
\Lambda_{1 i j}+\Lambda_{1 j k}+\Lambda_{1 k i}=d \Lambda_{0 i j k}
$$

If we now follow the same reasoning that was used to determine the value $2 \pi n$ for the cocycle condition in the study of $F_{2}$, we can find the periods of $\Lambda_{1 i j k} \equiv d \Lambda_{0 i j k}$ on any closed curve on the triple intersection where it has been defined. Considering an element $\Sigma_{3}$ of the 3-homology of $\mathcal{M}$ intersecting $U_{i}$, $U_{j}$ and $U_{k}$, and using formula (4.67) which states the condition on the periods of $F_{3}$ we obtain

$$
\int_{\Sigma_{3}} F_{3}=\int_{\Sigma_{1}} \Lambda_{1 i j k}=2 \pi n
$$


where $\Sigma_{1}$ is a closed curve on $U_{i} \cap U_{j} \cap U_{k}$. By construction $\Lambda_{1 i j k}$ is both closed and with integer periods in any closed curve in $U_{i} \cap U_{j} \cap U_{k}$ and therefore defines a uniform map $M$

$$
M: U_{i} \cap U_{j} \cap U_{k} \rightarrow U(1)
$$

At this point we find a new and interesting twist to the story. Since the transitions of the 2-forms are given by local one forms on the intersections of the elements of the covering, we can naturally build the following maps

$$
g:(i, j) \rightarrow g_{i j}(P, \mathcal{C}) \equiv \exp i \int_{\mathcal{C}} \Lambda_{1 i j}
$$

where $\mathcal{C}$ is an open curve with end points $O$ (a reference point) and $P . g$ associates to $(i, j)$ a map $g_{i j}(P, \mathcal{C})$ from the path space over $U_{i} \cap U_{j}$ to the group $U(1)$ and consequently, $g$ is a 1-cochain.

Notice that the 1-form $\eta_{i j}$ cannot be integrated out to obtain a transition function as in the case of a line bundle. However, if we apply Cech's coboundary operator to $g$ we obtain

$$
\delta g_{i j k}=g_{i j} g_{j k} g_{k i}=\exp i \int_{O}^{P} \Lambda_{1 i j k}
$$

which is in general different form the identity element of $U(1)$, and is in fact, the uniform map $M$ previously defined in (4.74). The fact that the coboundary $(4.76)$ is not the identity map on the group explicitly shows that the geometrical structure we are dealing with is not that of a $U(1)$ bundle. Nevertheless, (4.76) is a properly defined 2-cochain in Čech's cohomology 
theory. With this objectat hand, we may go a step furhter and consider the action of the coboundary operator $\delta$ on 2-cochains, and consequently we need to study what happens in the intersection of four open sets. We will show that a 2-cocycle can be built in such intersection. Indeed, in the intersection of four open sets $\left(U_{i} \cap U_{j} \cap U_{k} \cap U_{l}\right)$

$$
\begin{aligned}
d \Lambda_{0 i j k} & =\Lambda_{1 i j}+\Lambda_{1 j k}+\Lambda_{1 k i} \\
d \Lambda_{0 i j l} & =\Lambda_{1 i j}+\Lambda_{1 j l}+\Lambda_{1 l i} \\
d \Lambda_{0 i k l} & =\Lambda_{1 i k}+\Lambda_{1 k l}+\Lambda_{1 l i} \\
d \Lambda_{0 j k l} & =\Lambda_{1 j k}+\Lambda_{1 k l}+\Lambda_{1 l j}
\end{aligned}
$$

from where it follows that

$$
d\left(\Lambda_{0 i j k}-\Lambda_{0 i j l}+\Lambda_{0 i k l}-\Lambda_{0 j k l}\right)=0
$$

or equivalently,

$$
\Lambda_{0 i j k}-\Lambda_{0 i j l}+\Lambda_{0 i k l}-\Lambda_{0 j k l}=\text { constant }
$$

Using identity (4.73) we finally obtain,

$$
\Lambda_{0 i j k}-\Lambda_{0 i j l}+\Lambda_{0 i k l}-\Lambda_{0 j k l}=2 \pi n
$$

It is then natural to define the following 2-cochain on $U_{i} \cap U_{j} \cap U_{k}$

$$
g:(i, j, k) \rightarrow g_{i j k} \equiv \exp i \Lambda_{0 i j k}
$$


which does obviously satisfy the 2-cocycle condition

$$
\delta g_{i j k l}=g_{i j k} g_{i j l}^{-1} g_{i k l} g_{j k l}^{-1}=I
$$

We thus conclude that a 3-form with integer periods has an associated triplet $\left(A_{2}, \Lambda_{1}, \Lambda_{0}\right)$ of local forms, the latter of which satisfies a 3-cycle condition on the intersection of four open sets of the covering of the manifold, i.e. a 1-gerbe.

The procedure we have shown may be generalized to globally defined $p+1$-forms $F_{p+1}$ over $\mathcal{M}$, satisfying

$$
\begin{aligned}
d F_{p+1} & =0 \\
\oint_{\Sigma_{p+1}} F_{p+1} & =2 \pi n .
\end{aligned}
$$

This gives a geometrical structure with transition $p-1$ forms $\Lambda_{p-1}$ with values on the Lie algebra of the structure group leading to 1-cochains

$$
\exp i \int_{\Sigma_{p-1}} \Lambda_{p-1}
$$

$F_{p+1}$ being the field strenght (curvature) of a local $p$ form $A_{p}$ with transitions given by $d \Lambda_{p-1}$. Moreover on $U_{i} \cap U_{j} \cap U_{k} \neq \emptyset$ the $p-1$-transition form

$$
F_{p-2}=\Lambda_{p-1 i j}+\Lambda_{p-1 j k}+\lambda_{p-1 k i}
$$

satisfies the conditions

$$
\begin{aligned}
d F_{p-2} & =0 \\
\oint_{\Sigma_{p-2}} F_{p-2} & =2 \pi n
\end{aligned}
$$


and hence the structure of $F_{p-2}$ may be determined by induction. We end up with a $p$-cocycle condition on the intersection of $p+2$ open sets. Summing up, we have shown that the globally constrained $p+1$ form $F_{p+1}$ implies the existence existence of a tower (multiplet) of local antisymmetric fields with non trivial transition which in turn represents a $p$ gerbe.

In section 6, we will apply these lessons to show quantum equivalence of the $d=11$ supermembrane and the $d=10$ IIA Dirichlet supermembrane for the general case of non trivial line bundles associated to the $U(1)$ gauge fields in the Dirichlet supermembrane multiplet. We will thus extend previous proofs valid for trivial line bundles.

A last word about gerbes, as we stated in the introduction, gerbes only abelian gerbes have been developed into a full geometrical theory so far, while not much progress has been made in the non abelian case. The reasons for this do probably date back to the work of Teitelboim [17] who showed that non abelian theories for higher order forms do not exist. As we have seen in this section, the construction of gerbes in terms of multiplets of forms relies on a reasonable extension of Weil's theorem, a similar approach has been introduced in 18 19], to discus the construction of duality maps for non-abelian 2-forms but such work is still in progress. 


\section{Duality on $p$-Gerbes}

In this section, we discuss the general duality map relating local antisymmetric fields defining gerbes. The action for the local $U(1) p$-form $A_{p}$ defined over open sets of a covering of $\mathcal{M}$, a compact manifold of dimension $d \geq p+1$ with $p>1$, and with transitions given as in the previous section, is the following

$$
S\left(A_{p}\right)=\frac{1}{2} \int_{\mathcal{M}} F_{p+1} \wedge^{*} F_{p+1}+g_{p} \int_{\Sigma_{p}} A_{p}
$$

where $F_{p+1}$ is the globally defined curvature $p+1$-form associated to $A_{p} . \Sigma_{p}$ is a $p$-dimensional closed surface being the boundary of a $p+1$-chain. $g_{p}$ is the coupling associated to $A_{p}$. From (5.92) we obtain the field equations

$$
d^{*} F_{p+1}=g_{p} \delta_{\Sigma_{p}}
$$

where $\delta_{\Sigma_{p}}$ is the usual $d-p$-form associated to the Dirac density distribution.

Let us consider now the dual formulation to (5.92). Following the arguments of the previous sections, we introduce a constrained $p+1$-form $\Omega_{p+1}$ globally defined over $\mathcal{M}$ and satisfying

$$
\begin{aligned}
d \Omega_{p+1} & =0 \\
\oint_{\Sigma_{p}} \Omega_{p+1} & =\frac{2 \pi n}{g_{p}} .
\end{aligned}
$$

we also introduce the following action for $\Omega_{p+1}$

$$
I\left(\Omega_{p+1}\right) \equiv \frac{1}{2} \int_{\mathcal{M}} \Omega_{p+1} \wedge * \Omega_{p+1}+g_{p} \int_{\Sigma_{p+1}} \Omega_{p+1}
$$


where $\Sigma_{p+1}$ is a $p+1$-chain with boundary $\Sigma_{p}$.

The off-shell Lagrange problem of the above constrained system may be given by the extended action

$$
\mathcal{I}\left(\Omega_{p+1}, V_{d-p-2}\right)=S\left(\Omega_{p+1}\right)+i \int_{\mathcal{M}} \Omega_{p+1} \wedge W_{d-p-1}(V)
$$

where $W_{d-p-1} \equiv d V_{d-p-2}$ is the field strenght of the local $d-p-2$-form $V_{d-p-2}$, i.e. the curvature of $d-p-2$-gerbe. Consequently, $W_{d-p-1}$ identically satisfies the conditions

$$
\begin{aligned}
d W_{d-p-1} & =0 \\
\oint W_{d-p-1} & =\frac{2 \pi n}{g_{d-p-2}} .
\end{aligned}
$$

Integration on $V_{d-p-2}$ leads to action (5.92) while, integration on $\Omega_{p+1}$ yields the on-shell condition

$$
* \Omega_{p+1}=-i W_{d-p-1}-g_{p} \delta \Sigma_{p+1}
$$

and the dual action

$$
S\left(V_{d-p-2}\right)=\frac{1}{2} \int_{\mathcal{M}} W_{d-p-1}(V) \wedge * W_{d-p-1}+g_{d-p-2} \int_{\Sigma_{d-p-2}} V_{d-p-2}
$$

where

$$
\int_{\Sigma_{d-p-2}} \cdot=-\frac{g_{p}}{g_{d-p-2}} \int_{\mathcal{M}} d\left(* \delta\left(\Sigma_{p+1}\right)\right) \cdot
$$

From (5.93) and (5.100) we obtain the quantization condition

$$
g_{p} g_{d-p-2}=2 \pi n
$$


The quantum equivalence of the dual actions (5.92) and (5.101) follows once one integrates over all corresponding higher order bundles. This is a generalization of the equivalence proven in section (2) for the electromagnetic

duality. The quantization of charges is directly related to the different higher order bundles that may be constructed over $\mathcal{M}$ and it arises naturally from the global constraint (5.95) needed for having a well defined gerbe. The correspondence between closed integral p-forms and bundles is in general not one-to-one, depending on the topology of the base manifold, the redundancy being given by $H^{p-1}(\mathcal{M}, U(1))$ 20].

\section{Global analysis of duality maps in p-brane theories}

We use in this section the global arguments of the previous sections to improve the p-brane $\Leftrightarrow d$-brane equivalence that has been proposed by [21] [22] 23]. The duality transformation has been used by Townsend [21] to show the equivalence between the covariant $D=11$ supermembrane action with one coordinate $\left(x^{11}\right)$ compactified on $S^{1}$, and the fully $d=10$ Lorentz covariant worldvolumen action for the $d=10$ IIA Dirichlet supermembrane. The equivalence between the bosonic sectors was previously shown by Schmidhuber [23] using the Born-Infeld type action found by Leigh [22]. We will argue in a global way showing the equivalence between both theories, even when 
nontrivial line bundles are included in the construction of the D-brane action. We discuss later on the equivalence of the bosonic sectors when the coupling to background fields is included. Following the Howe-Tucker formulation of the $d=11$ supermembrane [21], we consider a supermembrane sitting on a target manifold with one coordinate compactified on $S^{1}$ [24], that is, we take $x^{11}$ to be the angular coordinate $\varphi$ on $S^{1}$, accordingly, we are interested in the following action

$$
\begin{aligned}
S & =-\frac{1}{2} \int_{X} d^{3} \xi \sqrt{-\gamma}\left[\gamma^{i j} \pi_{i}^{m} \pi_{j}^{n} \eta_{m n}+\right. \\
& \left.+\gamma^{i j}\left(\partial_{i} \varphi-i \bar{\theta} \Gamma_{11} \partial_{i} \theta\right)\left(\partial_{j} \varphi-i \bar{\theta} \Gamma_{11} \partial_{j} \theta\right)-1\right] \\
& -\frac{1}{6} \int_{X} d^{3} \xi \epsilon^{i j k}\left[b_{i j k}+3 b_{i j} \partial_{k} \varphi\right]
\end{aligned}
$$

where $\eta$ is the Minkoswski metric on a 10-dimensional spacetime, and

$$
\begin{aligned}
\pi^{m} & =d x^{m}-i \bar{\theta} \Gamma^{m} d \theta \\
\epsilon^{i j k} b_{i j k} & =3 \epsilon^{i j k}\left\{i \overline { \theta } \Gamma _ { m n } \partial _ { i } \theta \left[\pi_{i}^{m} \pi_{j}^{n}+i \pi_{i}^{m}\left(\bar{\theta} \Gamma^{n} \partial_{j} \theta\right)-\right.\right. \\
& \left.-\frac{1}{3}\left(\bar{\theta} \Gamma^{m} \partial_{i} \theta\right)\left(\bar{\theta} \Gamma^{n} \partial_{j} \theta\right)\right]+ \\
& \left.+\left(\bar{\theta} \Gamma_{11} \Gamma_{m} \partial_{i} \theta\right)\left(\bar{\theta} \Gamma_{11} \partial_{j} \theta\right)\left(\partial_{k} x^{m}-\frac{2 i}{3} \bar{\theta} \Gamma^{m} \partial_{k} \theta\right)\right\} \\
\epsilon^{i j k} b_{i j} & =-2 i \epsilon^{i j k} \bar{\theta} \Gamma_{m} \Gamma_{11} \partial_{i} \theta\left(\partial_{j} x^{m}-\frac{i}{2} \bar{\theta} \Gamma^{m} \partial_{j} \theta\right) .
\end{aligned}
$$

In order to discuss duality we will follow the approach we have introduced in the preceding sections. We begin by letting $\Sigma_{1}$ be a basis of homology on the three dimensional worldsheet manifold $(X), L_{1}$ be a 1 -form satisfying the 
constraints

$$
\begin{aligned}
d L_{1} & =0 \\
\oint_{\Sigma_{1}} L_{1} & =2 \pi n, \quad n \in \mathbb{Z}
\end{aligned}
$$

then, as we have learnt, $L_{1}$ defines a class of uniform maps $X \rightarrow S^{1}(-1$ gerbe) via

$$
\begin{aligned}
g & =\exp i \varphi, \quad \text { and } \\
L_{1} & =-i g^{-1} d g=d \varphi .
\end{aligned}
$$

The converse being also valid.

The next step in the construction of the duality map consists then in attaining an equivalent formulation to the action (6.104) in terms of the global 1-form $L_{1}$. In order to achieve this goal we must notice that the Lagrange formulation of the constraints on $L_{1}$ may be obtained in terms of a connection 1-form over the space of all non trivial line bundles, i.e. the global constraints can be included in the action by introducing an auxiliary line bundle with connection and coupling its curvature with $L_{1}$ (which we regard as globally defined but unconstrained) in the following fashion

$$
\int_{X} d F(A) \wedge L_{1}
$$

According to this, we begin the discussion of duality by introducing the 
following master action (here $L_{1 i}$ stands for the i-component of $L_{1}$ )

$$
\begin{aligned}
S_{\text {Master }} & =-\frac{1}{2} \int_{X} d^{3} \xi \sqrt{-\gamma}\left[\gamma^{i j} \pi_{i}^{m} \pi_{j}^{n} \eta_{m n}+\right. \\
& \left.+\gamma^{i j}\left(L_{1 i}-i \bar{\theta} \Gamma_{11} \partial_{i} \theta\right)\left(L_{1 j}-i \bar{\theta} \Gamma_{11} \partial_{j} \theta\right)-1\right]- \\
& -\frac{1}{6} \int_{X} d^{3} \xi \epsilon^{i j k}\left[b_{i j k}+3 b_{i j} L_{1 k}\right]+\int_{X} F(A) L_{1}
\end{aligned}
$$

Where $A$ is a connection on a line bundle over $X$ and where the path integral must sum over all connections and all line bundles.

Functional integration of the exponential of the master action on $A$ yields the following factor on the measure (recall the arguments in section 2)

$$
\delta\left(d L_{1}\right) \delta\left(\oint_{\Sigma_{1}} L_{1}-2 \pi n\right)
$$

We now use the fact that

$$
\delta\left(d L_{1}\right) \delta\left(\oint_{\Sigma_{1}} L_{1}-2 \pi n\right)=\int[d \varphi] \frac{\delta\left(L_{1}-d \varphi\right)}{\operatorname{det} d}
$$

where $\varphi$ defines a map from $X \rightarrow S^{1}$, this shows that $d \varphi$ satisfies the constraint on the periods. At this point we notice that the functional integral appearing in (6.115) is over all maps from $X \rightarrow S^{1}$, in other words, it is not an integration over a cohomology class defined by an element of $H^{1}(X)$. In distinction to section 2, the zero modes in this case, are constants. We may hence directly integrate the path integral associated to $S_{1}$ on $L_{1}$ and replace $L_{1}$ by $d \varphi$ a choice that leads us to the covariant $d=11$ supermembrane action. 
On the other hand, we might have functionally integrated over $L_{1}$ in to arrive to the functional integral of the action

$$
\begin{aligned}
S_{2} & =-\frac{1}{2} \int_{X} d^{3} \xi \sqrt{-\gamma}\left[\gamma^{i j} \pi_{i}^{m} \pi_{j}^{n} \eta_{m n}-\gamma^{i j} f_{i}(A) f_{j}(A)-1\right] \\
& -\frac{1}{6} \int_{X} d^{3} \xi \epsilon^{i j k} b_{i j k}+\int_{X} d^{3} \xi \gamma^{i j} f_{i}(A) i \bar{\theta} \Gamma_{11} \partial_{l} \theta
\end{aligned}
$$

Where

$$
f_{i}(A) \equiv \epsilon_{i m n}\left(F^{m n}(A)-\frac{1}{2} b^{m n}\right)
$$

The functional integral in $A$ must now be performed over all line bundles over $X$. The result (6.116) was obtained by Townsend in [21], for the case of a trivial line bundle. The equivalence between (6.116), the fully $d=10$ Lorentz covariant worldvolume action for the $d=10$ IIA Dirichlet supermembrane, and the $d=11$ covariant supermembrane action (6.104) has then been established. In the functional integral for (6.104), integration over all maps between $X \rightarrow S^{1}$ must be performed while in the functional integral for (6.116) the integration must be over the space of all connection 1-forms on all line bundles (modulo gauge transformations).

The global aspects of (6.116) are even more interesting when the coupling of the formulations to background fields is considered. In the $d=10$ membrane action obtained by dimensional reduction of the $d=11$ membrane theory the local 2-form $B$ of the NS-NS sector, couples to the current $\epsilon^{i j k} \partial_{k} \varphi$. The coupling is a topological one. Assuming we are in the euclidean worldvolume formulation of the theory, the coupling admits sources $B$ which are 
locally 2-forms but globally associated to nontrivial higher order gerbes. The reformulation of the action in terms of 1 -forms $L_{1}$ and constraints $d L_{1}=0$ and $\int L_{1} \sim n$ is close to what was done to obtain $S_{2}$ from the master action (6.113), but substituting $f_{i}(A)$ by

$$
\hat{f}_{i}(A) \equiv \epsilon_{i m n}\left(F^{m n}(A)+B^{m n}\right)
$$

where only the bosonic sector is considered. There is an interesting change in procedure, however, arising from the nontrivial transitions of $B$. The result is that $F^{m n}(A)$ must have also nontrivial transitions that compensate the ones of $B$. We have in the intersection of two opens $U^{\prime} \cap U \neq \phi$ where a nontrivial transition takes place

$$
\begin{aligned}
& B^{\prime}=B+d \eta \\
& F^{\prime}=F-d \eta
\end{aligned}
$$

which imply

$$
A^{\prime}=A-\eta
$$

This new transition for the connection 1-form A arises naturally in the topological field actions introduced in [25] to describe a gauge principle from which the Witten-Donaldson and Seiberg-Witten invariants may be obtained as correlation functions of the corresponding BRST invariant effective action. The most appropriate theory, however, where the nontrivial p-form connections are expected to have relevant non perturbative effects is the $d=11$ 
5-brane. It has been conjectured [21] that the $d=115$-brane action is given by

$$
S=-\frac{1}{2} \int_{X} d^{6} \xi \sqrt{-\gamma}\left[\gamma^{i j} \partial_{i} x^{M} \partial_{j} x^{N} \eta_{M N}+\frac{1}{2} \gamma^{i l} \gamma^{j m} \gamma^{k n} F_{i j k} F_{l m n}-4\right]
$$

where $F=d A$ is the self dual 3-form field strength of a local 2-form potential $A$, consequently, the context for this discussion is that of 1-gerbes. There is a very rich geometrical structure associated to this action with non perturbative effects related to the non trivial gerbes. The $d=115$-brane has been also interpreted [21] as a Dirichlet-brane of an open supermembrane, with boundary in the 5-brane worldvolume described by a new six-dimensional superstring theory previously conjectured by [26]. We expect that these intrinsic non-perturbative effects should be realized naturally over non-trivial higher order gerbes.

\section{Conclusions}

In this article we have introduced the notion of gerbes which are the natural setting to discuss $p$-forms with local transitions. We have also shown that gerbes allow a global extension of duality transformations in quantum field theory.

Our approach to duality incorporates globally constrained forms which give raise to the dual gerbes. The constraints can be easily incorporated into suitable master actions, care should be taken since the incorporation 
of the constraints has a subtlety associated with integration on boundaries i.e. stokes theorem. The constraints include relevant physical parameters such as coupling constants associated to the interaction of the $p$-forms to the underlying $p$-branes, or the radius of compactification of the superstring or supermembrane. The dependence becomes relevant in proving quantum equivalence between dual string and membrane theories.

Finally, we presented an improvement, of the equivalence between the covariant $d=11$ supermembrane action with one coordinate compactified on $S^{1}$ and the fully $d=10$ Lorentz covariant worldvolume action for the $d=10$

IIA Dirichlet supermembrane which even includes some global aspects.

\section{Acknowledgements}

M.C. would like to acknowledge the hospitality of the Center of Theoretical Physiscs (C.T.P.), Laboratory for Nuclear Science and Department of Physics of the Massachusetts Institute of Technology.

The financial support for this project came from several sources, we received partial funding from project $G-11$ form the Decanato de Investigaciones de la Universidad Simón Bolivar. The visit of M.I.C. to C.T.P. was made possible by a sabbatical grant form Universidad Simón Bolívar, and also by partial support from funds provided by the U.S. Department of Energy (D.O.E.) under cooperative research agreement DF-FCO2-94ER40810. 


\section{References}

[1] N. Seiberg and E. Witten, Nucl. Phys. B426 (1994) 19, B431 (1994) 484.

[2] S. Ferrara, J. Scherk and B. Zumino, Nucl. Phys. B121 (1977) 393;

E. Cremmer, S. Ferrara and J. Scherk, Phys. Lett. B74 (1978) 61; A. Ceresole, R. D'Auria and S. Ferrara, Phys. Lett.B339 (1994) 71; A. Sen, Int.J.Mod.Phys A9(1994) 3707; J.H.Schwarz and A. Sen, Nucl. Phys. B411 (1994) 35; I. Martin and A. Restuccia, Phys. Lett. B323 (1994) 311; M.J.Duff and J.X.Lu, Nucl. Phys. B426 (1994) 301; J.H. Schwarz, Lett. Math. Phys. 34 (1995) 309; M. Duff, Nucl. Phys. B442 (1995) 47; C. Hull and P. Townsend, Nucl. Phys. B438 (1995) 109; E. Witten, Nucl. Phys. B443 (1995) 85; A. Ceresole, R. D'Auria, S. Ferrara and A. van Proeyen, Nucl. Phys. B444 (1995) 92; E. Witten, hep-th /9507121. S. Kachru, A. Klemm, W. Lerche, P. Mayr and C. Vafa, Nucl. Phys. B459 (1996) 537 ; J. Stephany, Phys. Lett. B390 (1997) 128.

[3] E. Witten, hep-th/9505186; E. Verlinde, Nucl.Phys. B455 (1995) 211 ; Y. Lozano, Phys. Lett. B364 (1995) 19; A. Kehagias, hep-th/9508159.

[4] J.L.F. Barbón, Nucl. Phys. B452 (1995) 313

[5] J. Giraud, Cohomologie non-abelienne, volume 179 of Grundl. Springer Verlag, Berlin, 1997 
[6] J. L. Brylinski, Prog. in Math. Vol. 107, Loop Spaces Characteristic Classes and Geometric Quantization, Birkhäuser, Boston (1993).

[7] D. S. Chaterjee. On Gerbes, PhD Thesis, Cambridge University, 1998.

[8] P. Gajer. Geometry of Deligne Cohomology, Invent. Math., 127:155-207, 1997

[9] N. Hitchin Lectures on Special Lagrangian Submanifolds. In: School on Differential Geometry (1999), The Abdus Salam International Centre for Theoretical Physics. math.Dg/9907034

[10] R. Attal, math-ph/0203056.

[11] P. Gajer, Higher Holonomies, Geometric Loop Groups and Smooth Deligne Cohomology, in Advances in Geometry, pp. 195-236 vol 72 of Progress in Mathematics, 1999.

[12] M. Mackaay and R. Picken, math.DG/0007053

[13] P.G.O. Freund and R.I. Nepomechie, Nucl. Phys. B199 (1982) 482.

[14] R.I. Nepomechie, Phys. Rev. D31 (1985) 1921.

[15] B. Kostant, Lectures Notes in Mathematics, Berlin, Springer (1970) p.133.

[16] S. Eilenberg and N. Steenrod, Foundations of Algebraic Topology, Princeton, New Jersey, Princeton University Press (1964). 
[17] C. Teitelboim, Phys. Lett. 167B (1986) 63.

[18] I. Martín, hep-th/9905203

[19] I. Martín and A. Restuccia, hep-th/9703122.

[20] M. I. Caicedo, I. Martín and A. Restuccia, hep-th/9711122.

[21] P.K. Townsend, hep-th/9512062

[22] R. G. Leigh, Mod. Phys. Lett.A4 (1989) 2767.

[23] C. Schmidhuber, hep-th/9601003.

[24] P. Howe and R.W. Tucker, J. Phys.A10 (1977) 155; J. Math. Phys. 19 (1978) 981.

[25] R. Gianvittorio, I. Martin and A. Restuccia, Class. Q. Gravity, 13 (1996) 2887.

[26] E. Witten, hep-th/9507012 